\title{
The Effect of Gynostemma pentaphyllum Extract on Mouse Dermal Fibroblasts
}

\author{
Sara Nadia Lobo, Yu Qing Qi, and Quan Zhong Liu \\ Department of Dermatology, Tianjin Medical University General Hospital, International Students' Building, \\ Tianjin Medical University, Qixiangtai Road, No. 22, Heping District, Tianjin 300070, China
}

Correspondence should be addressed to Quan Zhong Liu; liuquanzhong@medmail.com.cn

Received 27 November 2013; Accepted 15 January 2014; Published 4 March 2014

Academic Editors: C. Feliciani and Y. Tuzun

Copyright (C) 2014 Sara Nadia Lobo et al. This is an open access article distributed under the Creative Commons Attribution License, which permits unrestricted use, distribution, and reproduction in any medium, provided the original work is properly cited.

Background. The objective of this paper is to demonstrate the effect of Gynostemma pentaphyllum extract on mouse dermal fibroblasts. Recent studies have shown that this plant may possess great antioxidant properties, which can be very beneficial in combating oxidative stress. Methods. Gynostemma pentaphyllum extract was prepared and mouse dermal fibroblasts were obtained and cultured as per our laboratory protocols. Twelve samples of cells were cultured under the same conditions and both negative and positive controls were established. Induction of oxidative stress was carried out using ultraviolet C (UVC) light. Viable cell count was carried out, using microscopy. The analysis of the overall results was processed using SPSS version 16.0. Results. Statistical analysis showed strong positive correlation between the concentration of Gynostemma pentaphyllum and the mean duration of cell viability $(\mathrm{rs}=1)$, with a high level of statistical significance $(P<0.01)$. Likewise, strong positive correlation existed between trials of cell viability $(\mathrm{rs}=0.988-1)$, with statistical significance $(P<0.01)$. Conclusion. Gynostemma pentaphyllum extract prolongs viability of mouse dermal fibroblasts damaged by UVC light-induced oxidative stress. The results show the potential benefits of this extract on dermal cell aging.

\section{Introduction}

The retardation and control of skin aging constitute one of the biggest challenges faced by researchers and scientists in the area of cosmetology. This area of study is continuously researched, due to the unending pursuit of maintenance of youthfulness and appearance. Many scientific investigations are currently underway, as the field of cosmeceutical development widens. New studies are revealing the truth about many agents that modify the process of skin aging. The future incorporation of these studies into clinical practice would change the way that this process is currently managed.

Many plant extracts play a vital role in the modification of skin aging. Some of these have been thoroughly investigated, whereas others have only been used empirically for centuries. Gynostemma pentaphyllum, commonly referred to as "Jiaogulan" in China, is one of the many examples of these plants $[1,2]$.
This plant is normally grown in the mountainous regions of China and is considered a vine because of its growth pattern. It has been used for centuries as a traditional remedy for respiratory and toxic conditions and has also been associated with longevity, among other benefits $[1,2]$.

Many studies have been done to verify some of the many beliefs associated with the use of this herb; however, as per the extensive research done prior to this proposal, no studies regarding the action of the plant extract on dermal fibroblasts have been documented or published.

The objective of this paper is to study the effects of Gynostemma pentaphyllum extract on dermal fibroblast, as it relates to skin aging. The hypothesis of this study is directed in favor of this plant extract, as being a significant contributor to the process of retardation of skin aging. This study has great scientific significance and will further contribute to the complete understanding of the benefits of this herb. This paper opens up the possibility for the development 
TABLE 1: Relationship between cell viability and exposure to UVC light.

\begin{tabular}{lc}
\hline UV light exposure $t / \mathrm{hrs}$ & Qualitative analysis of cell viability \\
\hline 0 & ++++ \\
0.5 & ++++ \\
1 & ++++ \\
1.5 & ++++ \\
2 & +++ \\
2.5 & ++ \\
3 & + \\
\hline
\end{tabular}

$\mathrm{L}++++$ all cells viable; +++: more viable cells than apoptotic cells; ++: more apoptotic cells than viable cells; +: total apoptosis.

of scientifically based cosmeceutical products, designed to combat the signs of skin aging.

\section{Materials and Methods}

(1) Preparation of Gynostemma pentaphyllum extract: $100 \mathrm{~mL}$ of double distilled water was heated to boiling point $\left(100^{\circ} \mathrm{C}\right) .100 \mathrm{mg}$ of the Gynostemma pentaphyllum herb was macerated and added to the double distilled water at boiling point. The mixture was then allowed to cool to room temperature. The solution was sterilized with 0.22 micrometer filters, sealed and subsequently stored at a $4^{\circ} \mathrm{C}$ in a refrigerator. A variety of different concentrations of the extract were subsequently obtained from the original solution.

(2) Mouse dermal fibroblasts were obtained and cultured as per protocols established by our Laboratory of the Department of Dermatology of the Tianjin Medical University General Hospital. Twelve samples of cells were cultured under the same conditions. One of the twelve samples was used as the positive control and was kept under optimum conditions throughout the entire experiment, without exposure to any aggressions or stimulants.

(3) The other eleven samples were placed under 8 wattsultraviolet C (UVC) light at a distance of $50 \mathrm{~cm}$ to induce oxidative stress. Induction of oxidative stress was limited to 1.5 hours.

(4) Subsequently, one of the eleven samples was placed under optimum conditions throughout the rest of the experiment, without any further exposure to aggressions or stimulants. This sample was considered as the negative control.

(5) The remaining ten samples were exposed to similar quantities of different concentrations of the Gynostemma pentaphyllum extract and, as with the rest of the samples, they were correctly labeled and placed under optimum conditions throughout the rest of the experiment, without any further exposure to aggressions or stimulants.

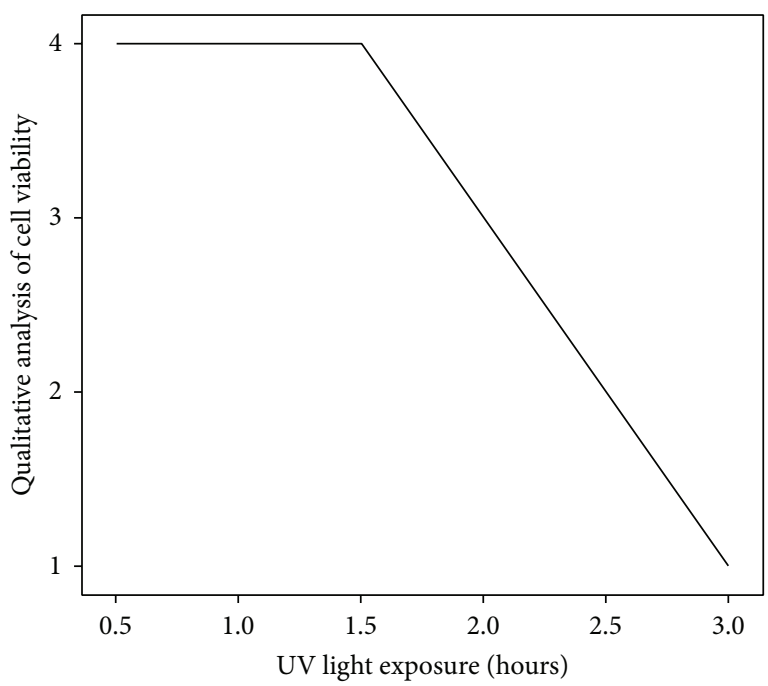

FIGURE 1: Relationship between cell viability and exposure to UVC light. For qualitative analysis of cell viability: 4-all cells viable; $3-$ more viable cells than apoptotic cells; 2 -more apoptotic cells than viable cells; 1 -total apoptosis.

(6) Qualitative analysis of cell viability was carried out using microscopy, as per our laboratory standards, at zero hours, followed by subsequent intervals.

(7) The entire procedure was officially repeated twice, for a total of three trials.

(8) Results obtained from the periodic observations were carefully and accurately documented.

(9) The analysis of the overall results was processed using SPSS version 16.0. Spearman's correlation coefficient was used to demonstrate the relationship between the variables used.

(10) The results were presented using charts and tables.

\section{Results}

UVC light induced a maximum level of oxidative stress at 1.5 hours of exposure (See Table 1 and Figure 1). After this period, signs of apoptosis were clearly visible (See Figures $4,5,6$, and 7). Qualitative analysis of cell viability revealed that an optimum concentration of Gynostemma pentaphyllum (taken as $4.5 \mathrm{mg} / \mathrm{mL}$ ) significantly prolonged the viability of cells exposed to UVC light (See Table 2 and Figures 2 and 3). Statistical analysis showed strong positive correlation between the concentration of Gynostemma pentaphyllum and the mean duration of cell viability $(\mathrm{rs}=1)$ with a high level of statistical significance $(P<0.01)$ (See Table 3(a)). Likewise, strong positive correlation existed between trials of cell viability $(\mathrm{rs}=0.988-1)$ with statistical significance $(P<$ 0.01) (See Table 3(b)).

\section{Discussion}

Gynostemma pentaphyllum belongs to the family of Cucurbitaceae and has been associated with the treatment of 
TABLE 2: Relationship between cell samples and duration of cell life.

\begin{tabular}{lccc}
\hline Duration of cell life/hrs & Negative control & Positive control & $\begin{array}{c}\text { Optimum concentration of } \\
\text { Gynostemma pentaphyllum }\end{array}$ \\
\hline 0 & ++++ & ++++ & ++++ \\
10 & + & ++++ & +++ \\
20 & + & ++++ & +++ \\
30 & + & ++++ & +++ \\
40 & + & ++++ & +++ \\
50 & + & +++ & + \\
60 & + & ++ & + \\
80 & + & + & +
\end{tabular}

$\mathrm{L}++++$ : all cells viable; +++: more viable cells than apoptotic cells; ++: more apoptotic cells than viable cells; +: total apoptosis.

TABLE 3: (a) Correlation between concentration of Gynostemma pentaphyllum and the mean duration of cell viability. (b) Correlation between trials of cell viability.

(a)

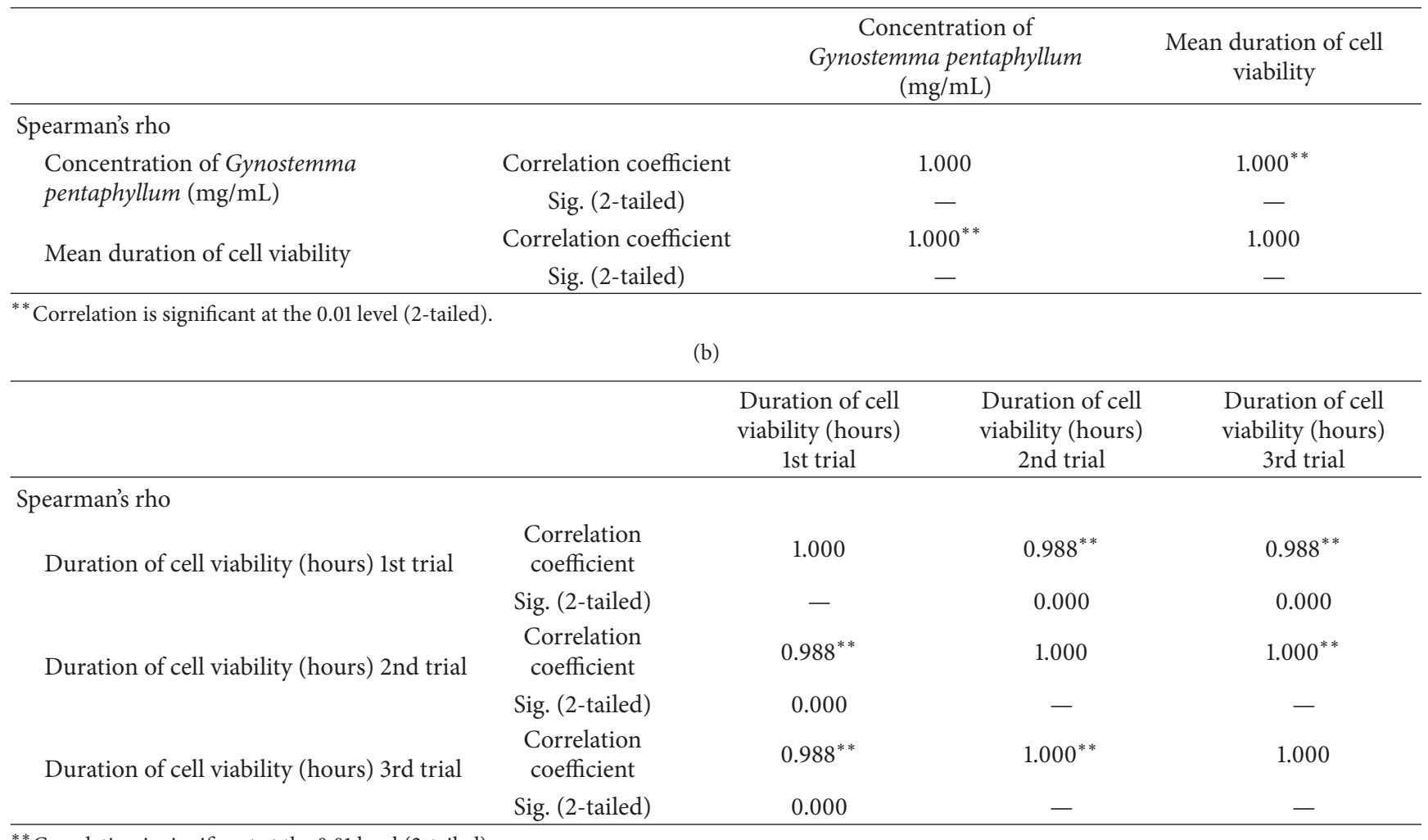

${ }^{* *}$ Correlation is significant at the 0.01 level (2-tailed).

cancer, gastric ulcers, hepatitis, respiratory diseases, and hyperlipidaemia, among other pathologies. One of the most recently discovered benefits of this herb is its antioxidant properties $[1,2]$.

The exact mechanisms involved in the pathophysiology of skin aging indicate that oxidative stress plays a vital role in the progress of this process. Any measures that can be taken to suppress the production of free radicals will facilitate the preservation of youthfulness of the skin. After thorough research on some of the naturally occurring antioxidants, Gynostemma pentaphyllum appears to be one of the most promising. Our results indicate that Gynostemma pentaphyllum is effective in prolonging the life of mouse dermal fibroblasts and that this effect increases in a dosedependent manner.

Many studies have been done to assert the potential of Gynostemma pentaphyllum as an antioxidant. The cells used in most of the studies vary widely, but the results are all statistically significant. The action of this plant extract on dermal fibroblasts has not been investigated, as per the 


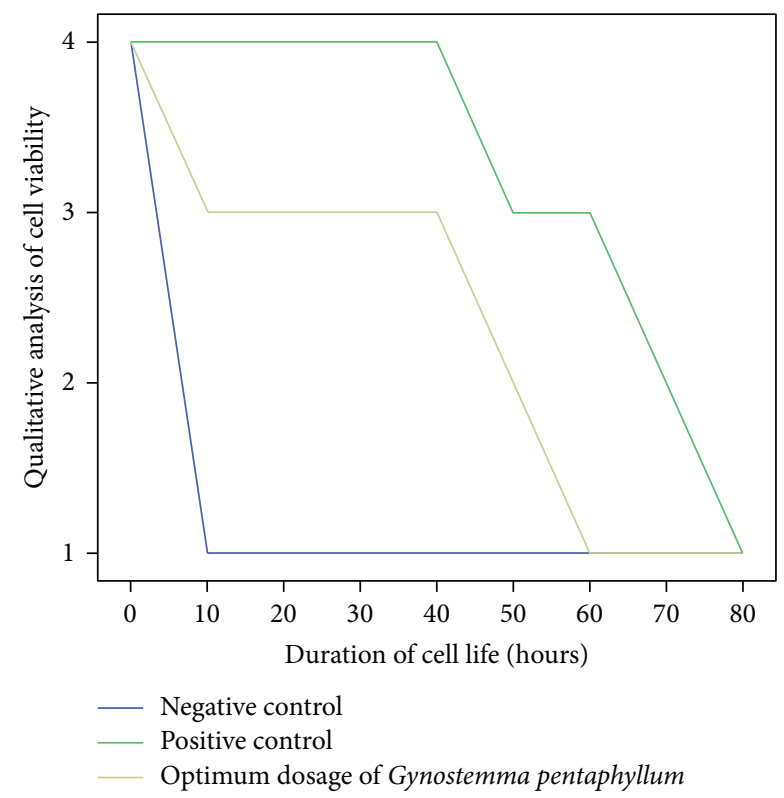

FIGURE 2: Relationship between cell samples and the duration of cell life. For qualitative analysis of cell viability: 4-all cells viable; $3-$ more viable cells than apoptotic cells; 2 -more apoptotic cells than viable cells; 1 - total apoptosis.

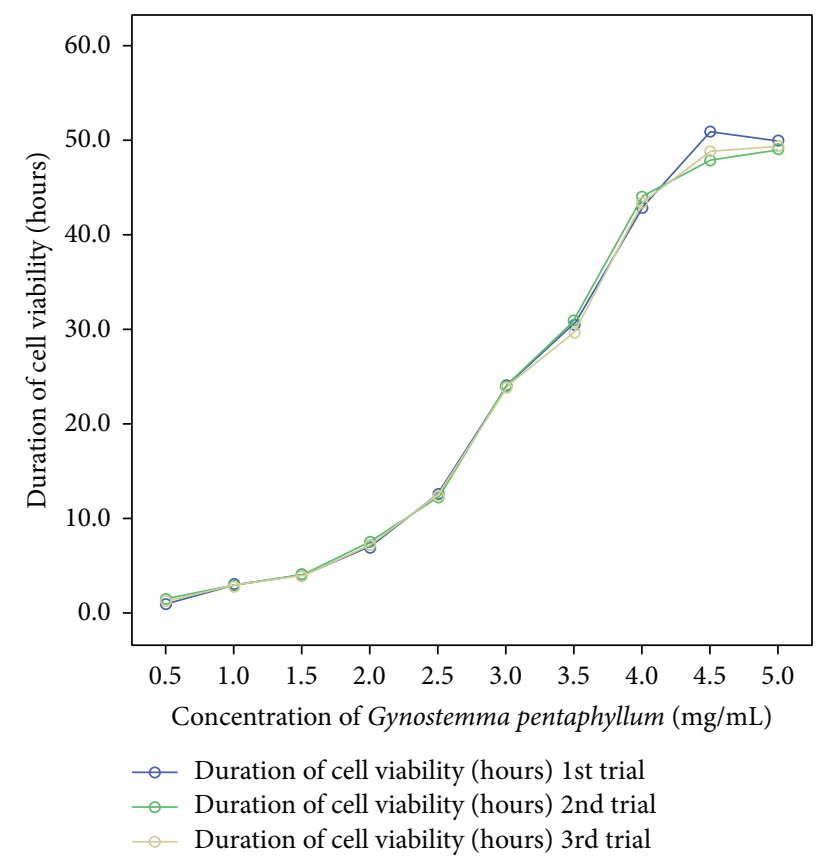

FIgURE 3: Relationship between cell viability and exposure to Gynostemma pentaphyllum following UVC light-induced oxidative stress.

research and review done. No studies are currently available to verify the effect of this extract on dermal fibroblast.

Schild et al. demonstrated the protective action of Gynostemma pentaphyllum extract on brain slices that were deprived of oxygen and glucose. This deprivation led to the onset of cell injury due to oxidative stress. Results showed

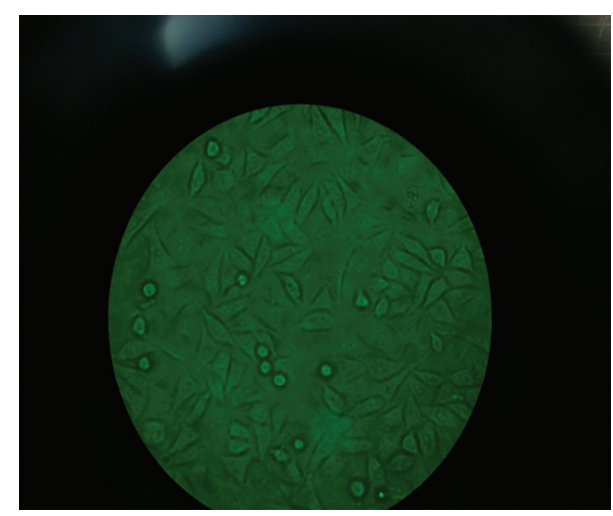

FIGURE 4: Photographs of various stages of the investigation (magnification 40x, no stain used). All cells viable.

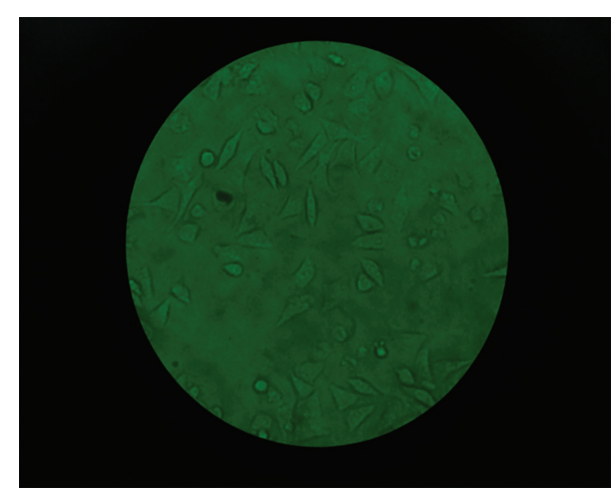

FIGURE 5: Photographs of various stages of the investigation (magnification 40x, no stain used). More viable cells than apoptotic cells.

that the use of this extract, within 48 hours of cultivation of cells, increased protein and activity levels of the antioxidative enzymes manganese superoxide dismutase (Mn-SOD) and glutathione peroxidase (GPx). Consequently, the cellular hydrogen peroxide $\left(\mathrm{H}_{2} \mathrm{O}_{2}\right)$ concentration remained at a low level. Similar antioxidant effects have been described in the findings of the study carried out by Zhang et al., who asserted the antioxidant properties of Gynostemma pentaphyllum extract on brain cells [3,4]. Likewise, significant favorable results have been obtained by different researchers who also investigated the protective antioxidant effect of Gynostemma pentaphyllum on brain cells $[5,6]$. Our results are consistent with those of the authors aforementioned, and these results validate the effectiveness of this extract in combating oxidative stress.

Müller et al. further contributed to studies regarding the antioxidant potential of this herb extract. The study was carried out using hepatocytes that were exposed to substances that induced oxidative stress. The extract completely protected the cells from oxidative stress-induced apoptosis. Similar results have been obtained by Lin et al. in their investigation using hepatocytes $[7,8]$.

Other studies done, using phagocytes and endothelial cells, such as that of Li et al., have produced significant results [9]. The fact that the action of this extract is nonspecific 


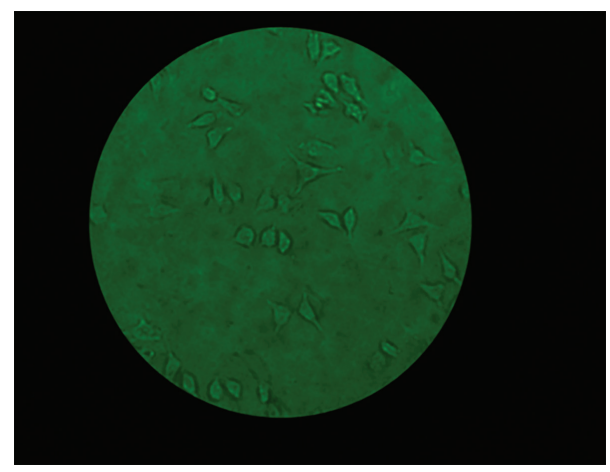

FIGURE 6: Photographs of various stages of the investigation (magnification 40x, no stain used). More apoptotic cells than viable cells.

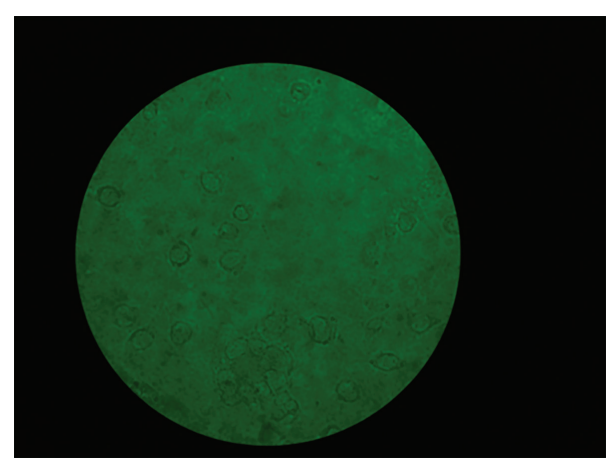

FIGURE 7: Photographs of various stages of the investigation (magnification 40x, no stain used). Total apoptosis.

created the base for pursuing our investigation. Our results now reveal that this extract is effective in prolonging the life of mouse dermal fibroblasts exposed to UVC light-induced oxidative stress.

Research done by Ma and Yang, at the Graduate School of the University of Science and Technology of Beijing demonstrated that Gynostemma pentaphyllum could scavenge active oxygen free radicals effectively [10]. Zhu et al. demonstrated in their study, using UV radiation, that Gynostemma pentaphyllum not only is a scavenger of free radicals but also possesses the biological function of anti-irradiation [11].

Other advantages of Gynostemma pentaphyllum have been discovered by other researchers. Gauhar et al. investigated the effect of Gynostemma pentaphyllum extract on obesity. This study was carried out at the preclinical level and has demonstrated significant results. The molecular mechanism of action involved the stimulation of fat oxidation and glucose uptake via AMPK activation in L6 myotube cells, with a corresponding decrease in body weight gain, liver weight, and blood cholesterol levels in mice [12].

In summary, our study shows that Gynostemma pentaphyllum is capable of reducing the effects of oxidative stress on mouse dermal fibroblasts. Oxidative stress accelerates aging and apoptosis. In our study, the prolongation of cell viability following UV induced oxidative stress served as a parameter for inferring that Gynostemma pentaphyllum extract acted as an antioxidant. This extract may significantly reduce the effects of oxidative stress on human dermal fibroblasts and may also reduce the aging and apoptosis of these cells. We carried out a qualitative assessment of mouse dermal fibroblast viability after exposure to UVC, in the presence and absence of Gynostemma pentaphyllum extract, as an initial phase in our ongoing research. Since no initial published results were available on this topic, we commenced our research with a qualitative, in vitro study. Given the fact that our results are promising, our team would proceed to perform a quantitative, preclinical study to corroborate our initial results. More research and investigation are required on this topic, in order to gain more insight into the actual mechanism of action of the extract on dermal cells. Our study also serves as a foundation on which new cosmeceutical products, such as antiaging and antioxidant creams and lotions, can be developed.

\section{Conclusion}

Our study reveals that Gynostemma pentaphyllum extract has a positive effect on the viability of mouse dermal fibroblasts that have been damaged by UVC light-induced oxidative stress. There is a very high level of association between the use of Gynostemma pentaphyllum and the prolonging of cell life. The potential repercussions of such an investigation are clearly visible, since this investigation creates a foundation for further studies in this field of dermatology. In addition, more profound research is required on this particular topic in order to establish valid comparisons between the results obtained and to isolate the actual compounds that are responsible for protecting the cells.

\section{Conflict of Interests}

The authors declare that there is no conflict of interests regarding the publication of this paper.

\section{Acknowledgments}

The authors of this investigation would like to acknowledge and extend their heartfelt gratitude to the Department of Dermatology and to the Laboratory of Dermatology of the Tianjin Medical University General Hospital, for providing the requisite materials, funding, and support throughout this investigation.

\section{References}

[1] W. C. Huang, M. L. Kuo, M. L. Li, R. C. Yang, C. J. Liou, and J. J. Shen, "The extract of Gynostemma pentaphyllum enhanced the production of antibodies and cytokines in mice," Yakugaku Zasshi, vol. 127, no. 5, pp. 889-896, 2007.

[2] C. Ulbright, Natural Standard Herb and Supplement Guide: An Evidence-Based Reference, Mosby Elsevier, St. Louis, Mo, USA, 2010.

[3] L. Schild, T. Cotte, G. Keilhoff, and R. Brödemann, "Preconditioning of brain slices against hypoxia induced injury 
by a Gynostemma pentaphyllum extract-stimulation of antioxidative enzyme expression," Phytomedicine, vol. 19, no. 8, pp. 812-818, 2012.

[4] G. L. Zhang, J. P. Deng, B. H. Wang et al., "Gypenosides improve cognitive impairment induced by chronic cerebral hypoperfusion in rats by suppressing oxidative stress and astrocytic activation," Behavioural Pharmacology, vol. 22, no. 7, pp. 633-644, 2011.

[5] L. Shang, J. Liu, Q. Zhu et al., "Gypenosides protect primary cultures of rat cortical cells against oxidative neurotoxicity," Brain Research, vol. 1102, no. 1, pp. 163-174, 2006.

[6] P. Wang, L. Niu, L. Gao et al., "Neuroprotective effect of gypenosides against oxidative injury in the substantia nigra of a mouse model of parkinson's disease," Journal of International Medical Research, vol. 38, no. 3, pp. 1084-1092, 2010.

[7] C. Müller, A. Gardemann, G. Keilhoff, D. Peter, I. Wiswedel, and L. Schild, "Prevention of free fatty acid-induced lipid accumulation, oxidative stress, and cell death in primary hepatocyte cultures by a Gynostemma pentaphyllum extract," Phytomedicine, vol. 19, no. 5, pp. 395-401, 2012.

[8] C. C. Lin, P. C. Huang, and J. M. Lin, "Antioxidant and hepatoprotective effects of anoectochilus formosanus and gynostemma pentaphyllum," The American Journal of Chinese Medicine, vol. 28, no. 1, pp. 87-96, 2000.

[9] L. Li, L. P. Jiao, and B. H. S. Lau, "Protective effect of gypenosides against oxidative stress in phagocytes, vascular endothelial cells and liver microsomes," Cancer Biotherapy, vol. 8, no. 3, pp. 263272, 1993.

[10] Z. Ma and Z. Yang, "Scavenging effects of Astragalus and Gynostemma pentaphyllum with its product on $\mathrm{O} 2-$. and .OH," Zhong Yao Cai, vol. 22, no. 6, pp. 303-306, 1999.

[11] S. Zhu, C. Fang, S. Zhu, F. Peng, L. Zhang, and C. Fan, "Inhibitory effects of gynostemma pentaphyllum on the UV induction of bacteriophage $\lambda$ in lysogenic Escherichia coli," Current Microbiology, vol. 43, no. 4, pp. 299-301, 2001.

[12] R. Gauhar, S. L. Hwang, S. S. Jeong et al., "Heat-processed Gynostemma pentaphyllum extract improves obesity in ob/ob mice by activating AMP-activated protein kinase," Biotechnology Letters, vol. 34, no. 9, pp. 1607-1616, 2012. 


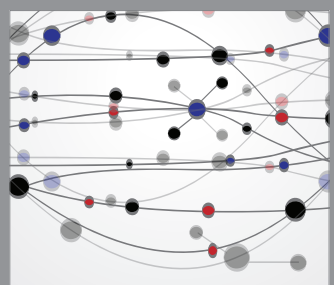

The Scientific World Journal
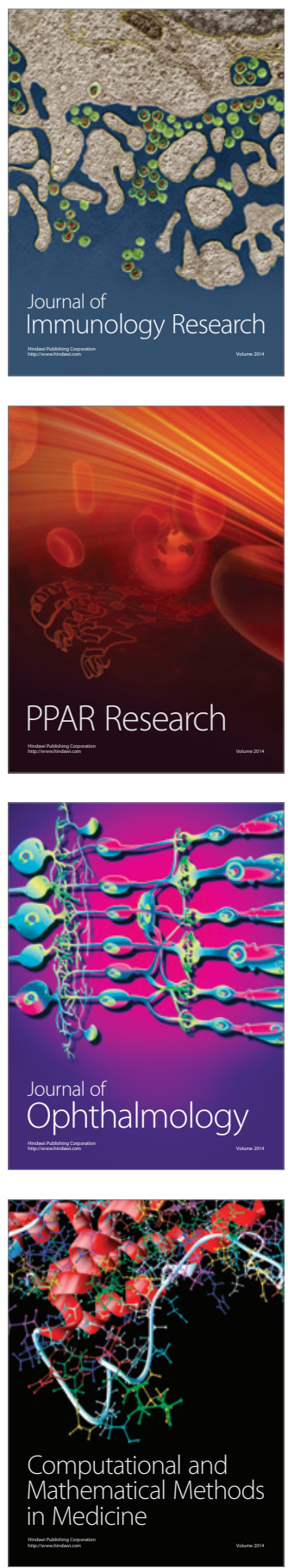

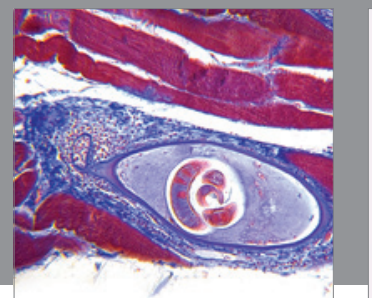

Gastroenterology

Research and Practice
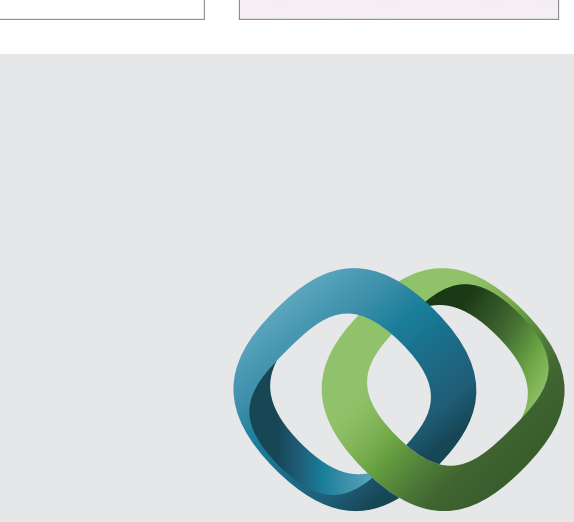

\section{Hindawi}

Submit your manuscripts at

http://www.hindawi.com
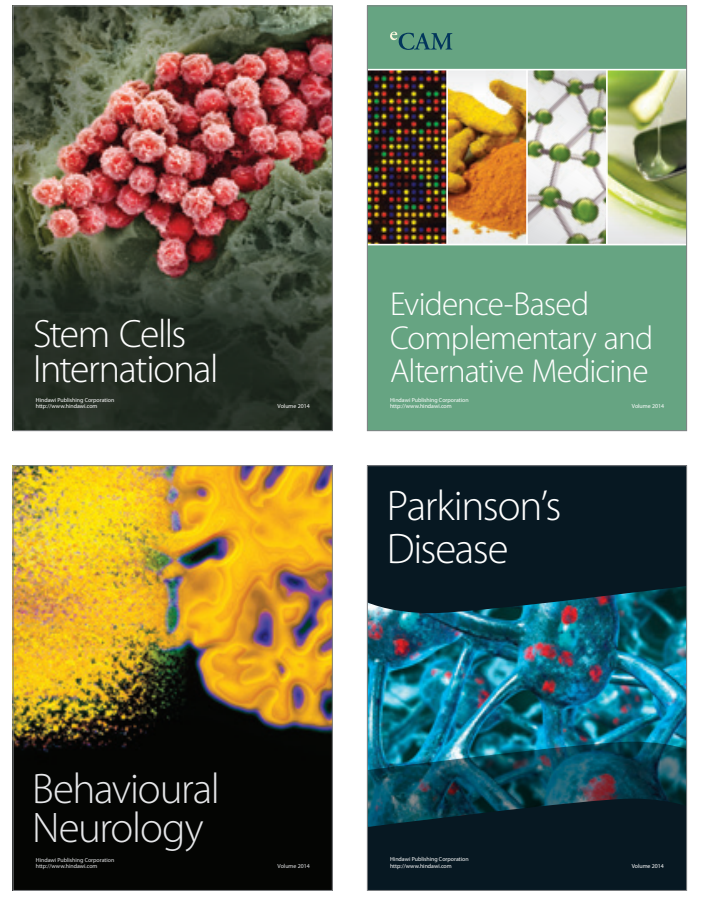
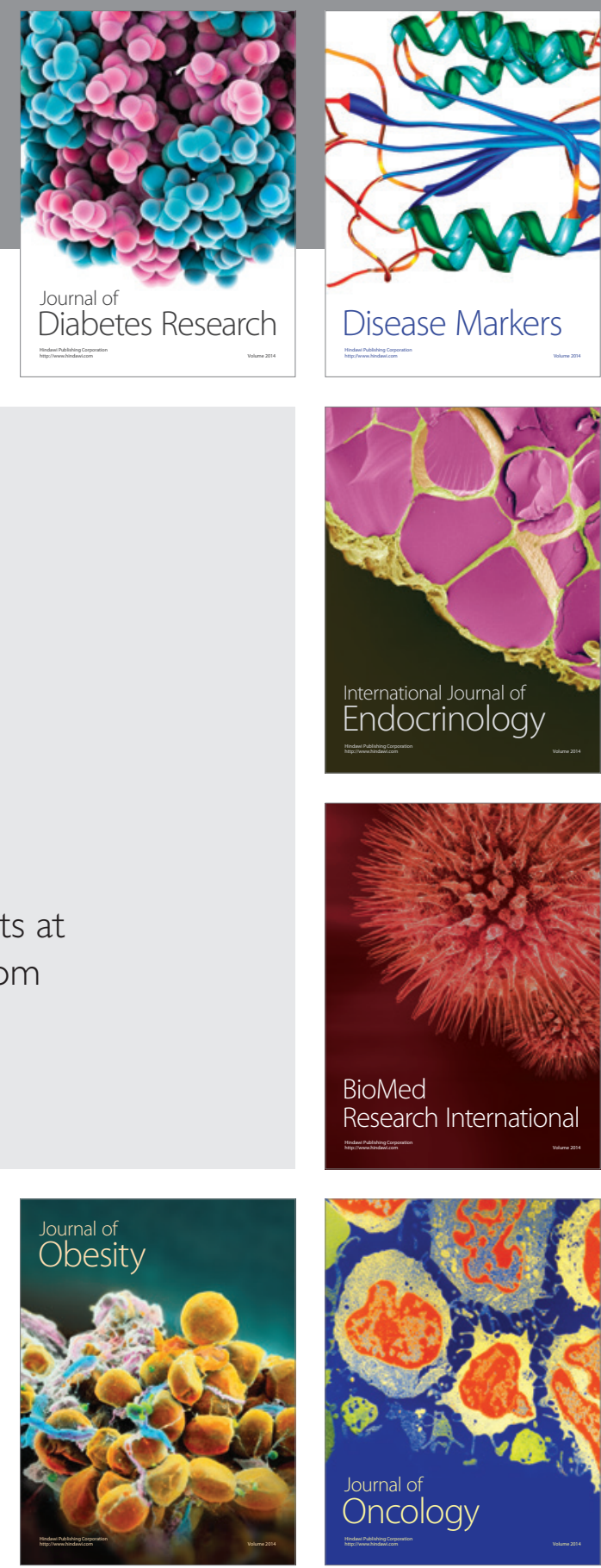

Disease Markers
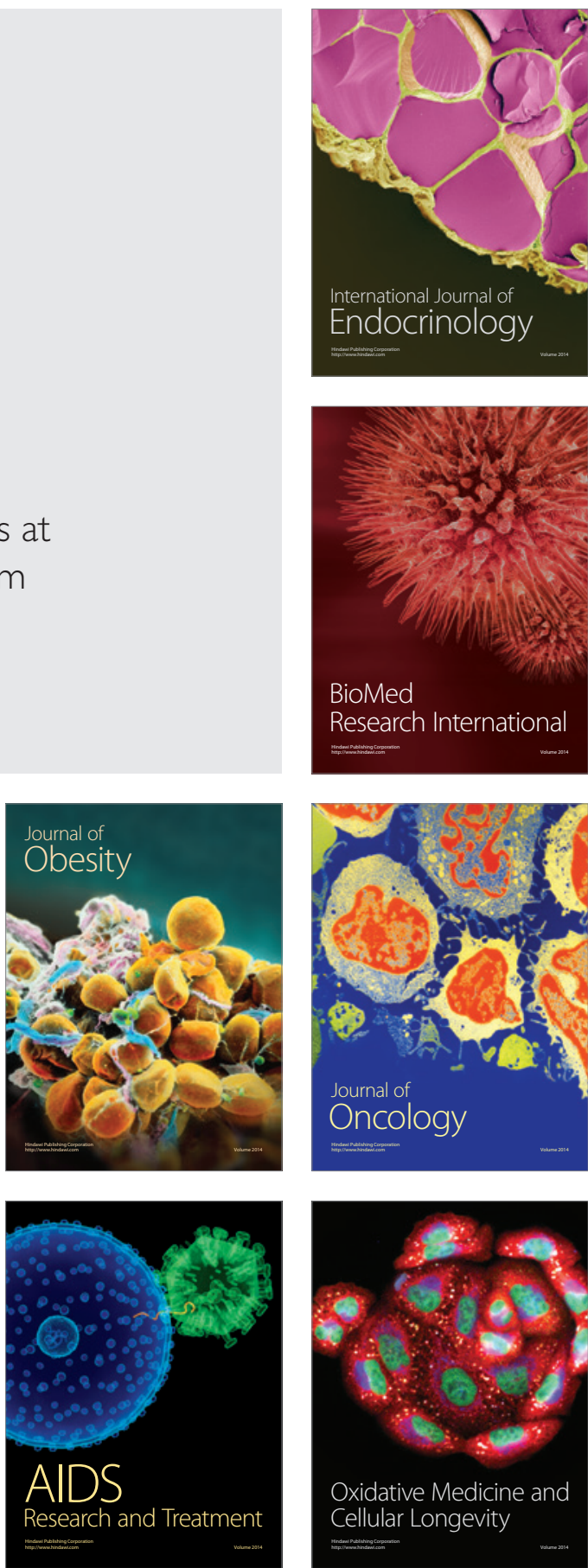